\title{
Comparison Control Strategies for ISG hybrid electric vehicle
}

\author{
Hailu Tang ${ }^{1, a}$ \\ School of Automotive Engineering,Wuhan University of Technology, Wuhan Hubei 430070, China \\ atanghailu0712@163.com,
}

Keywords: Hybrid electric vehicle; ISG; ADVISOR; control strategy.

Abstract. The paper takes Honda Insight parallel hybrid electric vehicle (HEV) as the research subject, which is assisted by an integrated starter generator. Apply the Insight vehicle simulation model in the ADVISOR software, and formulates the Rule-based Control Strategy (RBCS) and the Fuzzy Logic Control Strategy (FLCS) in the MATLAB/SIMULINK environment based on the Backward Simulation principle. In order to embed the two control strategies into the operating system of ADVISOR, the paper redeveloped the ADVISOR2002 for the off-line simulation of the two control strategies. Compared with the vehicle's performance under the two control strategy, the validity of the FLCS was verified.

\section{Introduction}

Faced with the energy, environment, climate and other aspects of the challenge, people turn their attention to hybrid electric vehicle (HEV). Because of outstanding fuel efficiency, low emission pollution, rapid start-stop control, power-assisted resistance, the use of renewable energy, the integrated starter-generator integrated (ISG) technology become one of the key technologies of parallel hybrid electric vehicle.

To develop HEV, control strategy, motor and battery are composed of three key technologies ${ }^{[1]}$. At present, HEV control strategy is mainly divided into four types: Rule-based control strategy (RBCS), Instantaneous optimization for $\mathrm{HEV}(\mathrm{IOH})$, Global optimization for $\mathrm{HEV}$ in particular cycle fuel consumption $(\mathrm{GOH})$, Adaptive predictive control strategy (APCS). The algorithm of RBCS is simple, and easy to realize real-time embedded system, which has good robustness ${ }^{[2]}$. However, it cannot achieve the optimal allocation of energy, which is developed for the specific conditions. So it is less portable, and can't adapt dynamic change condition ${ }^{[2]}$. IOH can realize the control of the specific steps, with a good adaptability, but cannot guarantee that it's the best in the whole operating range. Although $\mathrm{GOH}$ can really achieve the optimization, the algorithm of the control strategy is more complex, with a large amount of calculation, which is difficult to be applied in real life ${ }^{[2]}$. APCS needs traffic flow information to predict the condition of road provided by the vehicle GPS system and ITS, and DP algorithm is applied to get the global optimal solution in predicting front road conditions.

Therefore, in this paper a fuzzy logic rule based control strategy is proposed for a HEV. The demand torque and the battery SOC are input, the engine torque as the output to construct fuzzy controller, and determine the optional torque distribution between engine and motor, maximum improving the overall energy conversion efficiency of the system.

\section{The design of the control strategy}

\section{Rule-based Logic threshold Control Strategy}

This paper adopts Honda Insight power system as mild hybrid system, and chooses $1.0 \mathrm{~L}, 41 \mathrm{~kW}$ engine and $10 \mathrm{~kW}$ motor. And develop the multi energy control strategy based on logic threshold value. Through the torque limit module, torque calculation module and SOC correction module, Logic threshold control strategy distribute the torque coming from torque coupling device to make the engine work in the high efficiency region economy, and control the battery charge state in a certain range. The flow chart and the top module of control strategy as shown in Figure 1, Figure 2: 


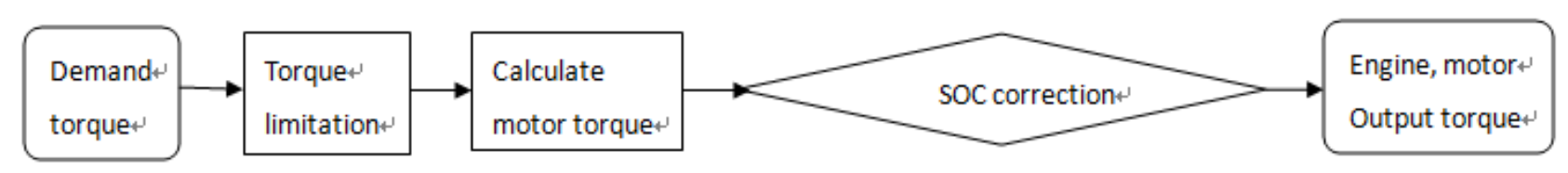

Figure 1. Control strategy flow chart

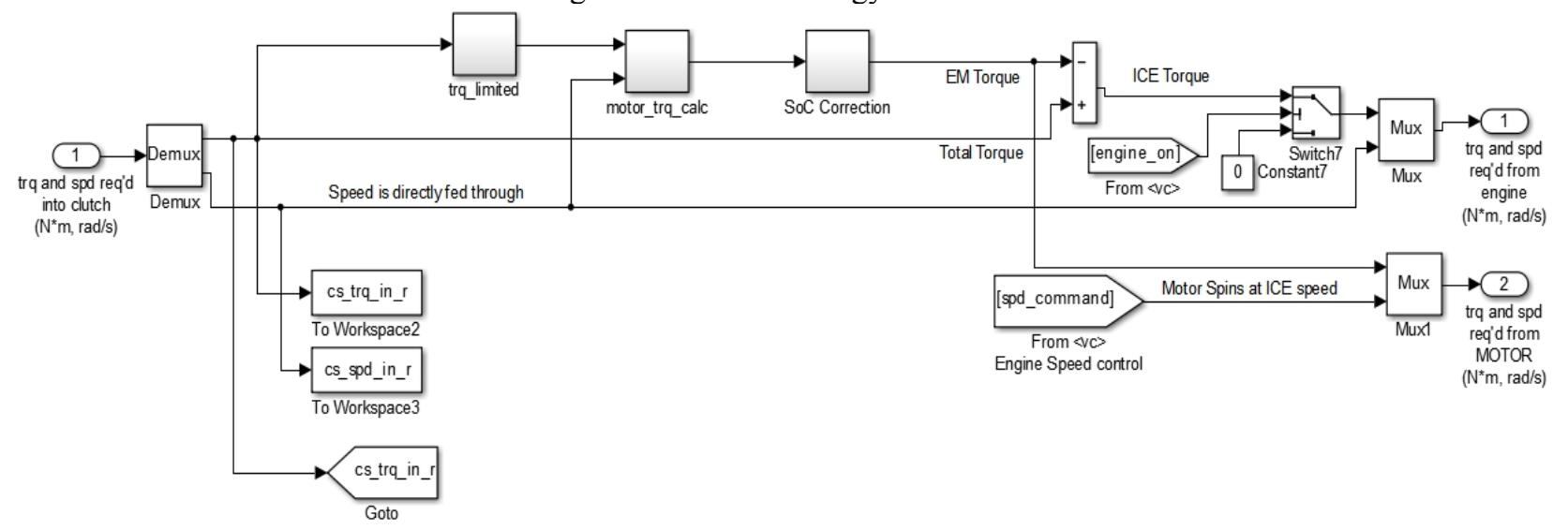

Figure 2. Control strategy top module.

\section{Fuzzy Logic Control Strategy}

Design of the fuzzy torque controller includes the following aspects:(1) decide the fuzzy controller input and output; (2) calculate the membership function of the fuzzy variables; (3) determine the fuzzy control rules; (4) derive outputs control variable of fuzzy torque controller. Figure 3 is the schematic diagram of fuzzy controller:

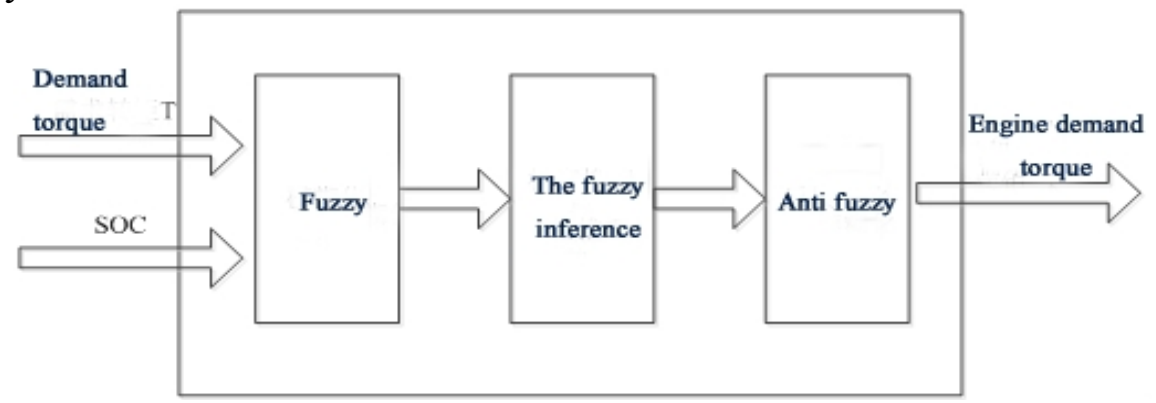

Figure 3. The schematic diagram of fuzzy controller

\section{A. Input, output and membership function}

According to the aim of fuzzy torque controller design and the efficiency of the engine MAP, the input variables of fuzzy torque controller are determined as: dynamic coupled vehicle demand torque $\mathrm{T}$ and battery state of charge SOC. The output variables of the fuzzy controller are the engine demand torque command Tr. The vehicle's demand torque $\mathrm{T}$ is divided into five subsets: $\{\mathrm{N}$ (negative), NM (negative medium), Z (zero), PM (positive medium), P (positive) $\}$, the domain is defined in the [1, 11], formula:

$$
\begin{cases}\frac{5 T}{T_{\text {eff }}}+1, & T \leq T_{\text {eff }} \\ \frac{5\left(T-T_{\text {eff }}\right)}{T_{\text {max }}-T_{\text {eff }}}+6, & T>T_{\text {eff }}\end{cases}
$$

where $\mathrm{T}_{\mathrm{gff}}=$ optimal engine torque curve at current speed; $\mathrm{T}_{\max }=$ the maximum output torque of engine at current speed.

Similarly, the battery SOC is divided into 5 subsets according to the range of SOC. Five fuzzy subsets: $\{\mathrm{L}($ low $), \mathrm{LM}$ (low medium),M(moderate),H(high medium),H(high) $\}$, the domain is defined in the $[1,11]$, formula: 


$$
\frac{10 \times(S O C-0.8)}{0.8-0.6}+11
$$

The engine output torque is divided into five subsets: $\{-2,-1,0,1,2\}$, domain defined in $[1,11]$.

The requirement of input torque of fuzzy torque controller, battery SOC value and the output torque of the engine expects membership function is shown in figure 4.

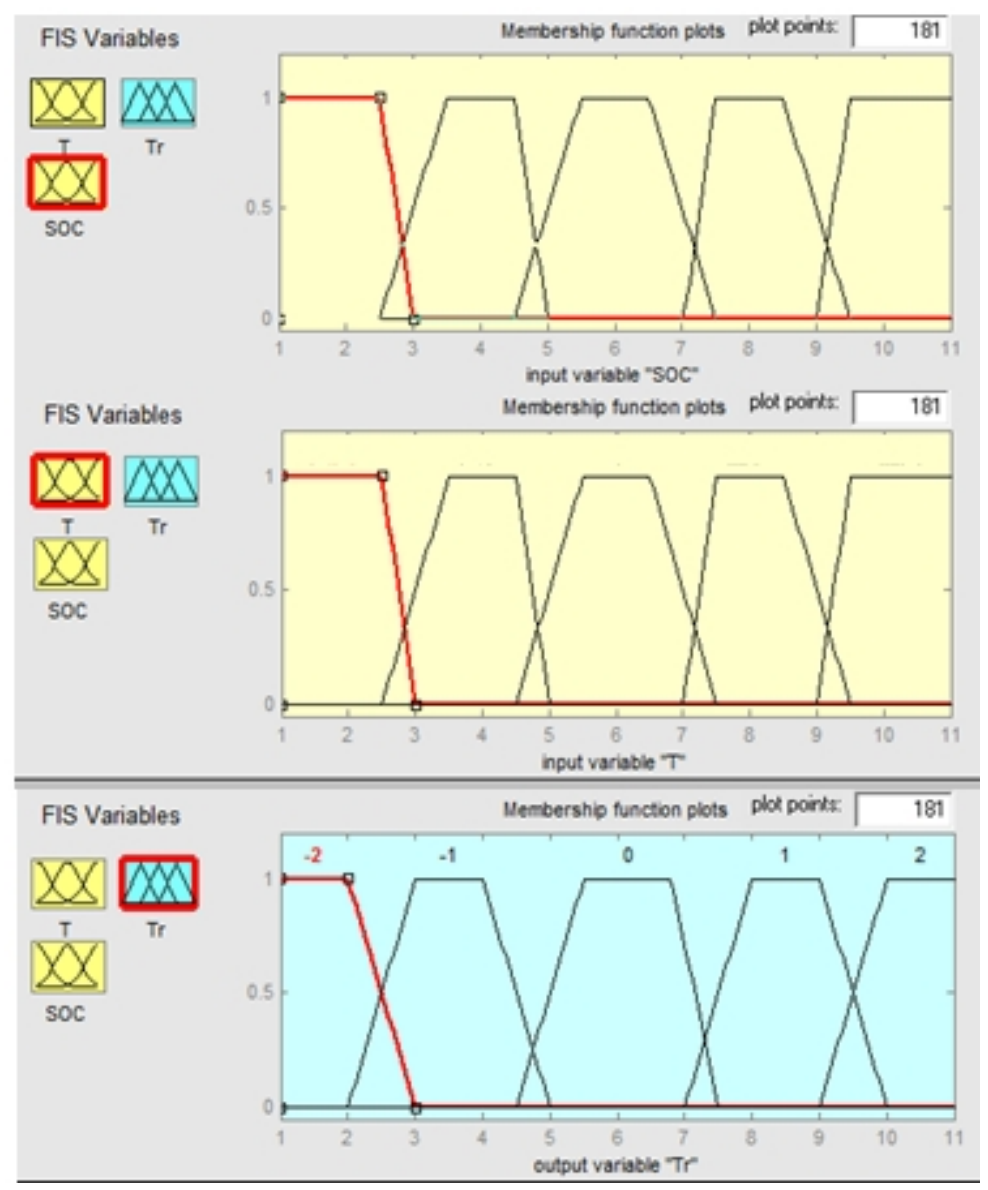

Figure 4. The membership function of FLC

\section{B. The rules of fuzzy control}

FLCS is based on the understanding of the object physical characteristics and the experience of the project. The basic control rule of FLCS is: the demand torque and battery SOC as input, and the engine torque as the output, FLCS has formulated the corresponding fuzzy control rules, which principle is as far as possible to make the engine work in the high efficiency, low fuel consumption, and maintain the battery state of charge in a certain value fluctuation.

According to the condition, establish the rule library "IF-THEN", and a fuzzy logic control table are given in Table 1.

Table 1. Fuzzy control rules

\begin{tabular}{c|ccccc}
\hline TOC & N & NM & Z & PM & P \\
\hline L & 0 & 0 & 1 & 1 & 2 \\
LM & -1 & 0 & 0 & 0 & 1 \\
M & -2 & -1 & -1 & -1 & 0 \\
HM & -2 & -2 & -2 & -2 & -1 \\
H & -2 & -2 & -2 & -2 & -1 \\
\hline
\end{tabular}




\section{Simulation results}

Simulation technology plays an important role in the design of electric vehicle, which not only can shorten the design cycle, reduce development costs, but also improve the performance of the car. The proposed control strategy is embedded in the vehicle model of ADVISOR, containing Insight models, where the backward simulation is carried out in the control panel.

\section{Two kinds of simulation results}

\section{A. Rule-based Control Strategy simulation results}

Combine the logic threshold control strategy with ADVISOR Insight vehicle model for off-line simulation. The strategy is tested by simulation with the UDDS cycle, and the initial SOC value is set to 0.7, the simulation results figures:

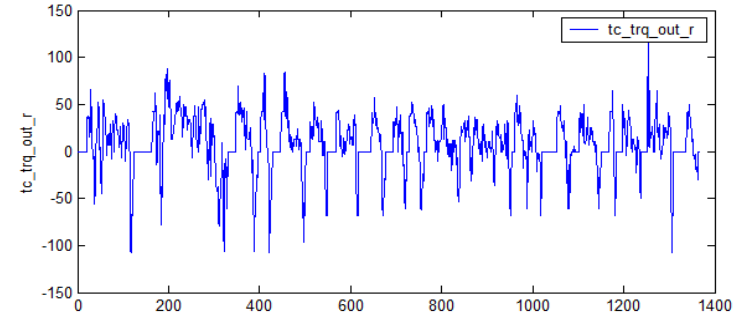

Figure 5. The demand torque.

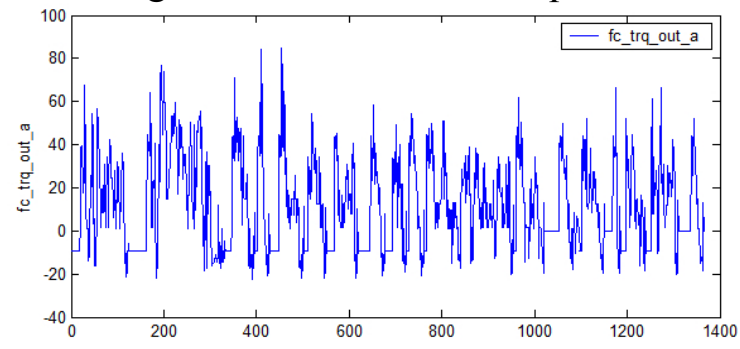

Figure 6. RBCS engine output torque.

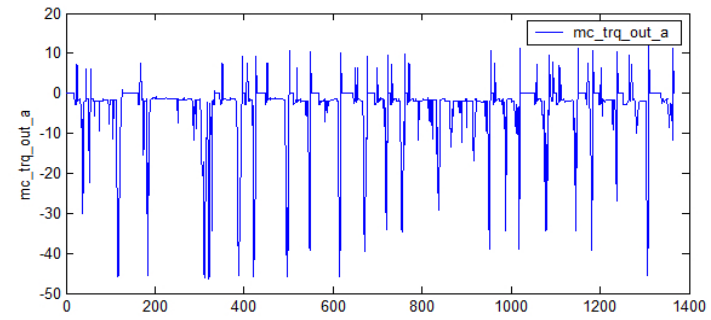

Figure 7. RBCS motor output torque.

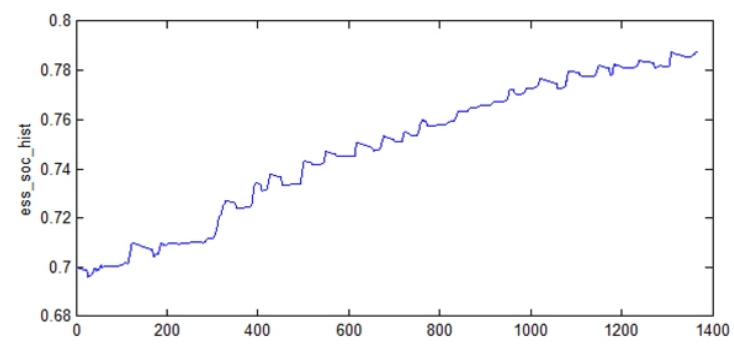

Figure 8. BSC changes of SOC cell
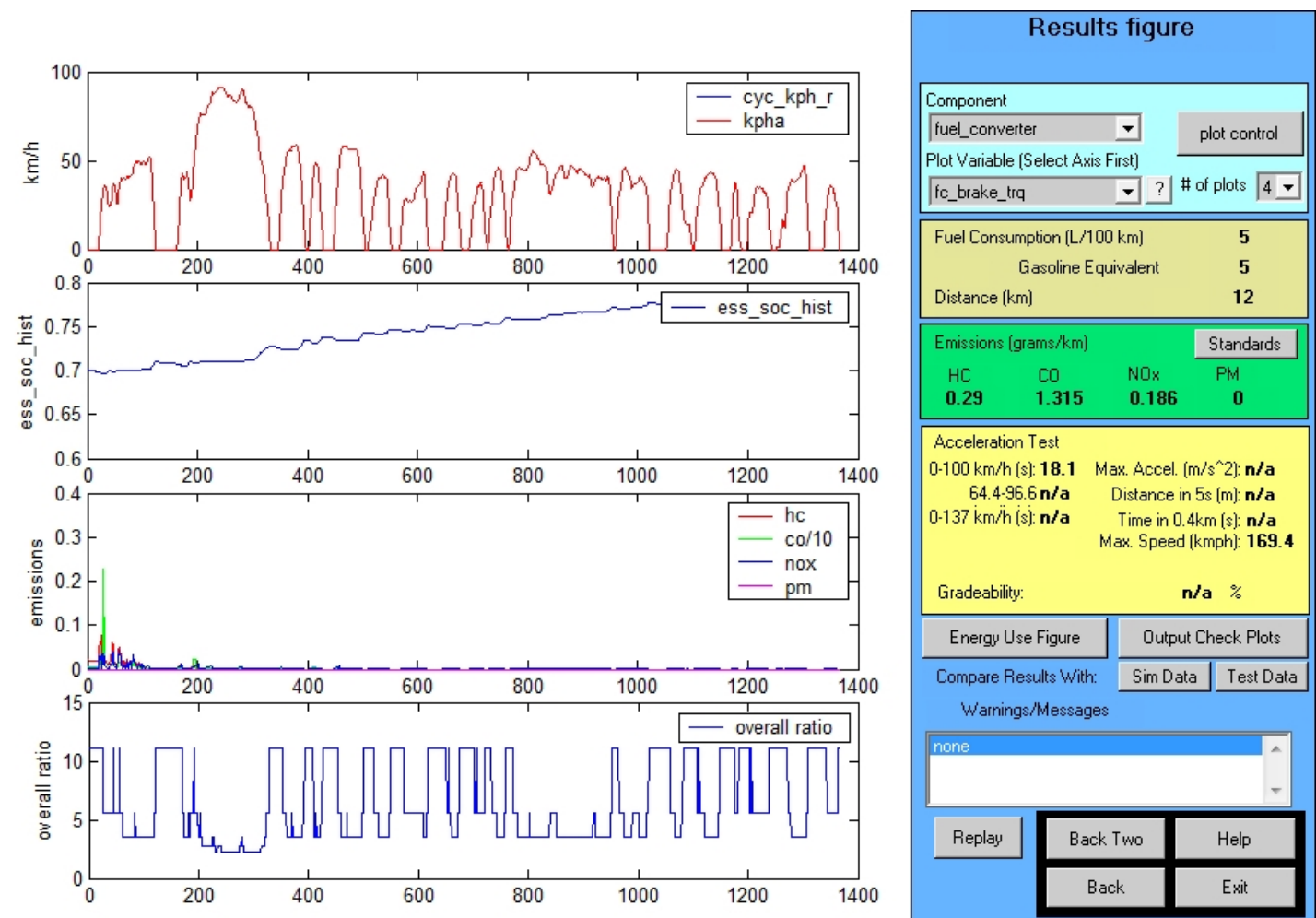

Figure 9. RBCS simulation result output interface 


\section{B. Fuzzy Logic Control Strategy simulation results}

Combine the FLCS with ADVISOR Insight vehicle model for off-line simulation. The strategy is tested by simulation with the UDDS cycle, and the initial SOC value is set to 0.7 , the simulation results figures:

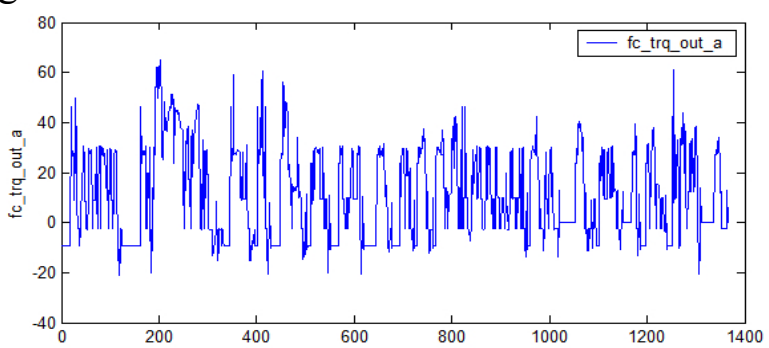

Figure 10. FLCS engine output torque

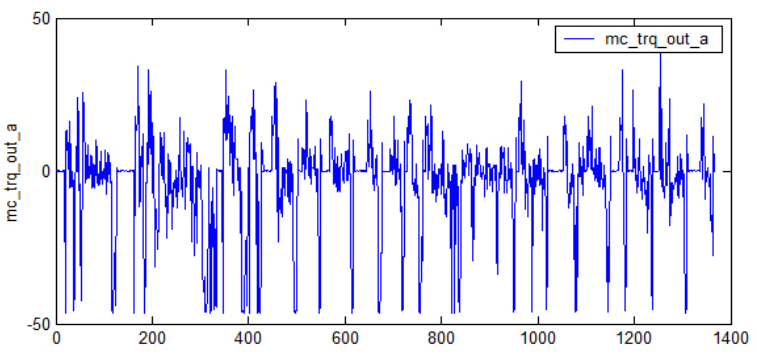

Figure 11. FLCS motor output torque

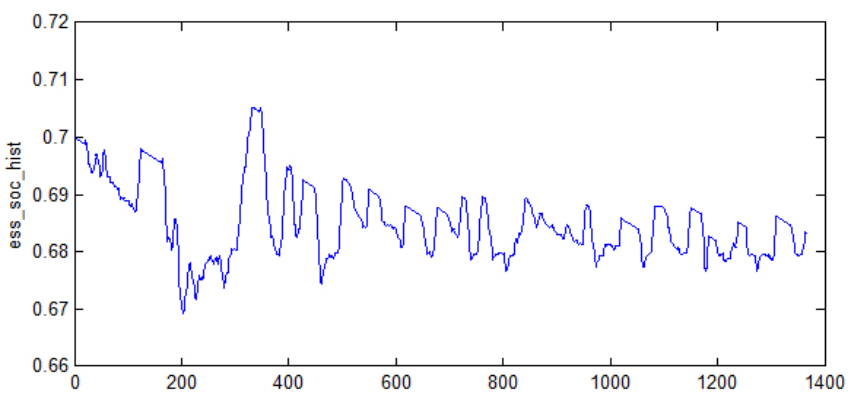

Figure 12. FLCS changes of SOC cell
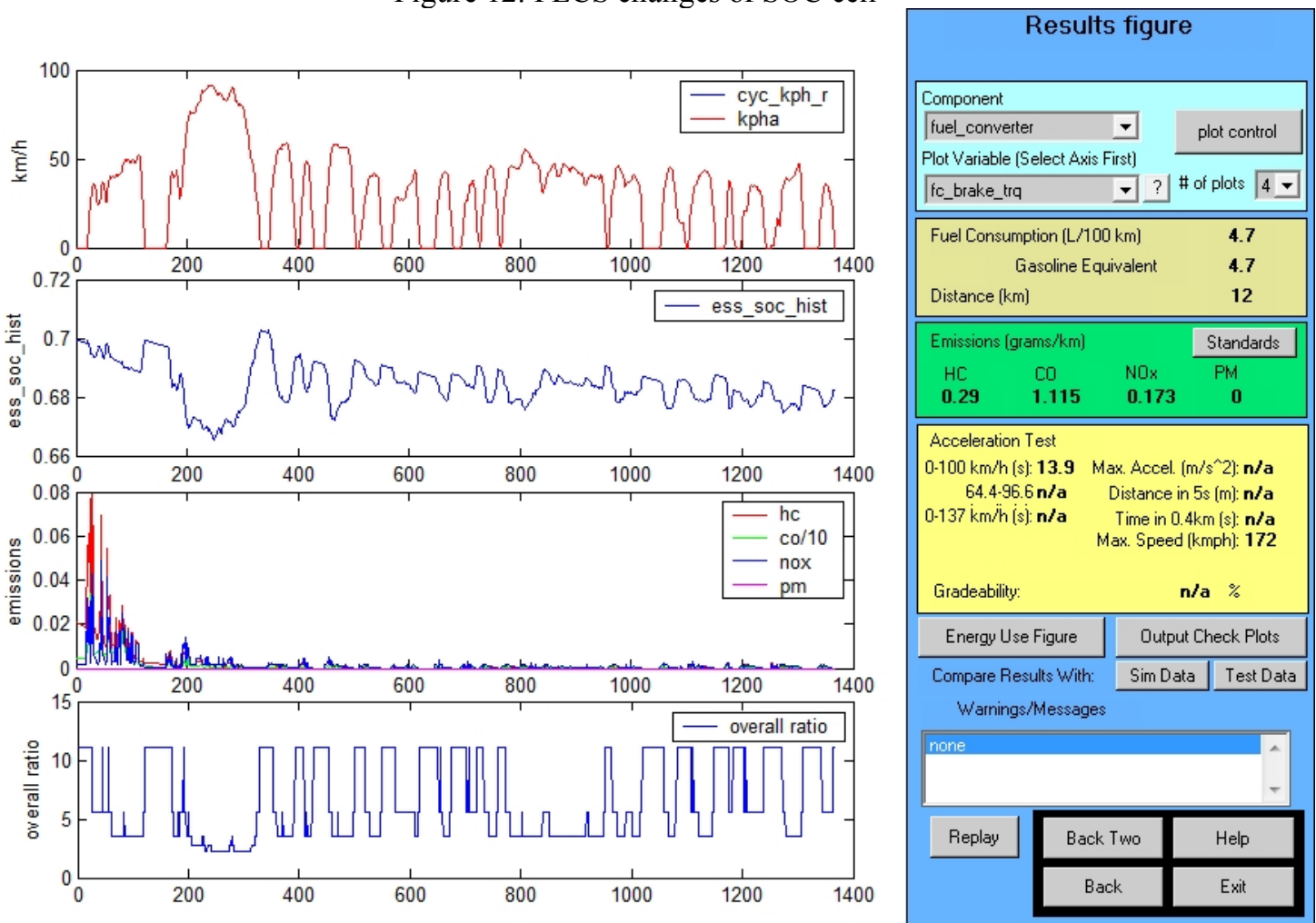

Figure 13. FLCS simulation result output interface.

Figure 5, 6, 7, 10, 11 show when the load demand is positive, majority power comes from engine and motor supplies parts of the supporting force while battery SOC meet the situation requirement; however when the load demand is negative and battery SOC is less than the limit maximum, motor mainly supplies braking force and parts coming from engine, recovering braking energy at the same time, which meets its dynamic performance requirement.

Figure 8 shows the maximum value of the SOC battery is about 0.786 , and the minimum value is about 0.698 , floating in 0.087 , the cycle remained at about 0.75 . 
Figure 12 shows the maximum value of the SOC battery is about 0.705 , and the minimum value is about 0.665 , floating in 0.040 , the cycle remained at about 0.7 . Both meet the requirements of the service life of the battery.

As described in Figure 9 and figure 13, two kinds of control strategy meet the Design original intention of energy saving and emission reduction.

\section{Comparison between Rule-Based and Fuzzy Logic Control Strategy}

According to the simulation results, two kinds of control strategy will be compared next.

Figure 14,15 show the engine working point distribution in the vicinity of $18 \%$ with the RBCS, scattered in the high efficiency area. However, with the fuzzy control strategy, the engine operating point moves to the high overall area, and the distribution is more concentrated, generally around $28 \%$. In the aspect of control the engine working in the high efficient area, FLCS is more effective.

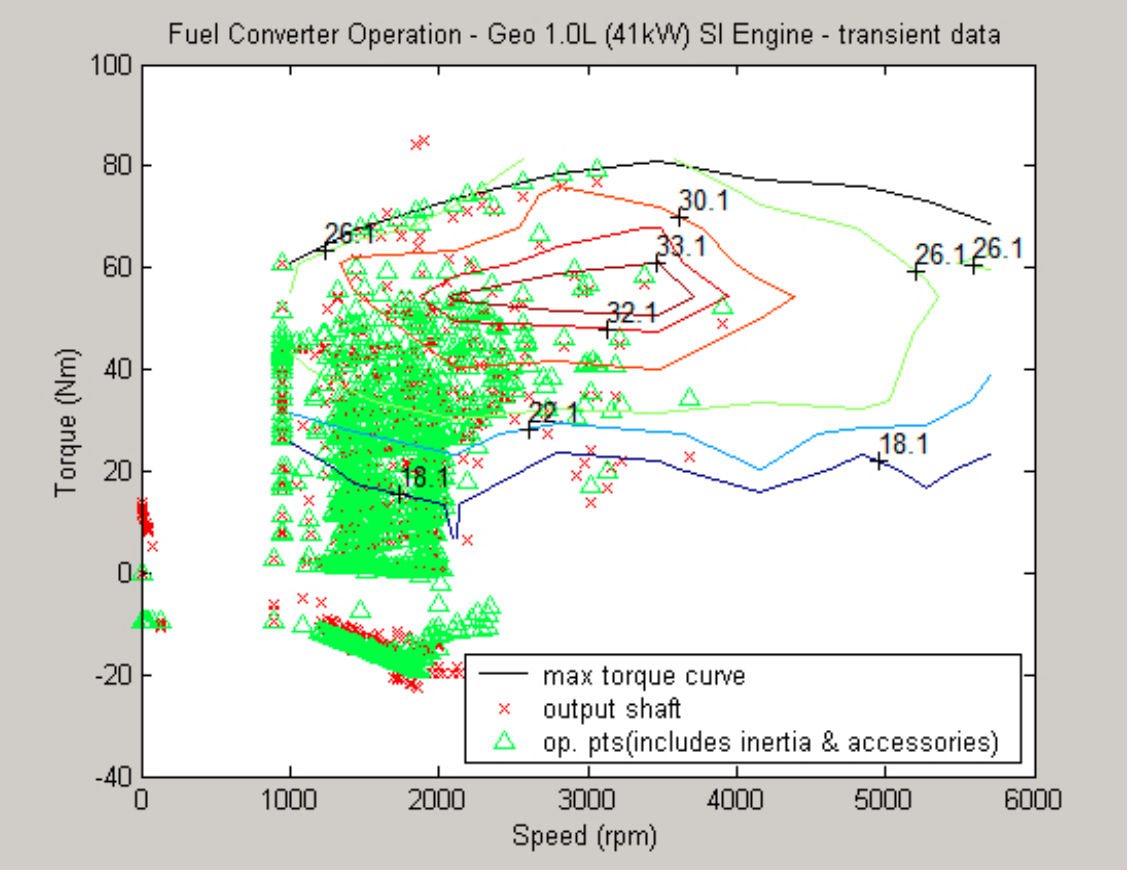

Figure 14. RBCS engine working area.

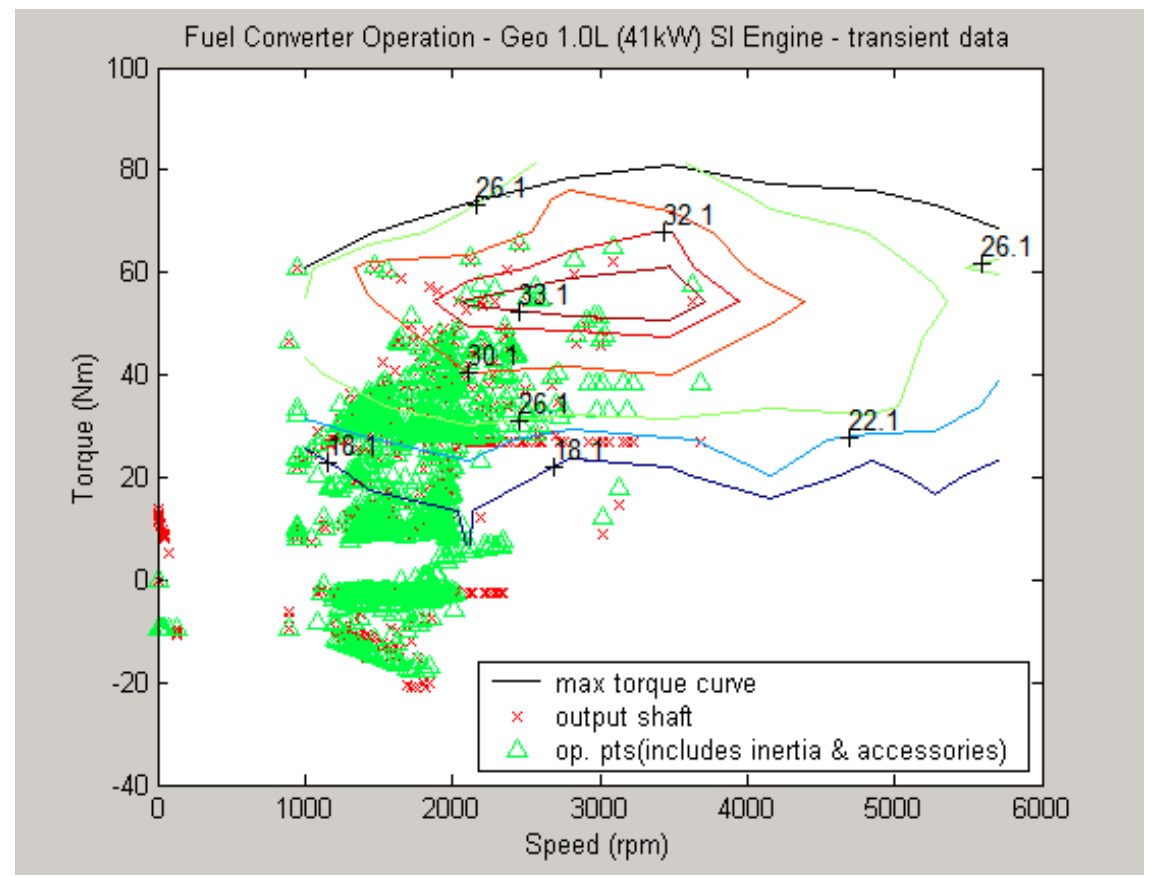

Figure 15. FLCS engine working area 
Compared with RBCS, some significant rules can be seen clearly from table 2 with FLCS.

(1) Fuel economy:

The fuel consumption per hundred kilometers decreased the $0.3 \mathrm{~L} / 100 \mathrm{~km}$, and saving rate was $6 \%$, improved fuel economy.

(2) Acceleration Performance:

0 to $100 \mathrm{~km} / \mathrm{h}$ acceleration time reduced $4.2 \mathrm{~s}$, optimizing the $23 \%$, and making a great improvement in the RBCS, which is special suitable for the vehicle frequent start stop running in urban road. And the maximum speed also improved $1.5 \%$, while the rate of increase is not great. But for the vehicle in urban road, they rarely reach the maximum speed of the vehicle, so the factor is little impact. Therefore, the Acceleration Performance of the vehicle with the FLCS get greatly improved, special suitable for the vehicle driving in city road.

(3) Emissions:

The emission of CO and NOX reduced by $0.197 \mathrm{~g} / \mathrm{km}, 0.01 \mathrm{~g} / \mathrm{km}$ and decreasing ratios were $15 \%$ and $5 \%$ respectively, reducing the emission of pollutants.

Table 2. Simulation results of two kinds of control strategy.

\begin{tabular}{ccccc}
\hline \multicolumn{2}{c}{ vehicle performance } & RBCS & FLCS & $\begin{array}{c}\text { Improvement } \\
\text { ratio }\end{array}$ \\
\hline Fuel economy & $\mathrm{Q}_{\mathrm{s}}(\mathrm{L} / 100 \mathrm{~km})$ & 5.0 & 4.7 & $6 \%$ \\
Acceleration & $0-100 \mathrm{~km} / \mathrm{h}$ time $(\mathrm{s})$ & 18.1 & 13.9 & $23 \%$ \\
Performance & $\mathrm{u}_{\mathrm{amax}}(\mathrm{km} / \mathrm{h})$ & 169.4 & 172.0 & $1.5 \%$ \\
& $\mathrm{HC}(\mathrm{g} / \mathrm{km})$ & 0.29 & 0.29 & $0 \%$ \\
& $\mathrm{CO}(\mathrm{g} / \mathrm{km})$ & 1.315 & 1.115 & $15 \%$ \\
& $\mathrm{NO} \mathrm{X}_{\mathrm{X}}(\mathrm{g} / \mathrm{km})$ & 0.186 & 0.173 & $5 \%$ \\
\hline
\end{tabular}

\section{Conclusions}

This paper formulates the Rule-based Control Strategy and the Fuzzy Logic Control Strategy. In order to embed the two control strategies into the operating system of ADVISOR, the paper redeveloped the ADVISOR2002 for the off-line simulation of vehicle performance with the two control strategies. Through the analysis of simulation results, found that the car with fuzzy control strategy is better on fuel economy, lower emissions of pollutants.

\section{References}

[1] Wei Hanbing, He Yituan, Li Jun, Deng Tao. Classification, State of the Art and Development Tendency of Strategies for Hybrid Electric Vehicles[J]. Journal of Chongqing Jiaotong University(Natural Sciences),2013,32(5).

[2] Wei Hanbing, He Yituan, Li Jun, Deng Tao. Classification, State of the Art and Development Tendency of Strategies for Hybrid Electric Vehicles[J]. Journal of Chongqing Jiaotong University(Natural Sciences),2013,32(5).

[3] Markel T, Brooker A.ADVISOR: A Systems Analysis Tool for Advanced Vehicle Modeling[J]. Journal of Power Source, 2002, 4801:1-12.

[4] Jeffery McGehee, Hwan-Sik Yoon. An Optimal Powertrain Control Strategy for a Mild Hybrid Electric Vehicle[C]. SAE International, 2013

[5] [20] Yu Zhisheng. Automobile theory [M]. Beijing: mechanical industry publishing house, 2009. 